\title{
Emisión de metano en ovinos alimentados con Pennisetum purpureum y árboles que contienen taninos condensados
}

\author{
Enteric methane emission in sheep fed Pennisetum purpureum \\ and tropical trees containing condensed tannins
}

\author{
Angel Trinidad Piñeiro-Vázquez*ad, Jorge Rodolfo Canul-Solis ${ }^{a}$, Fernando Casanova-Lugo ${ }^{\text {, }}$ Alfonso Juventino \\ Chay-Canulc, Armin Javier Ayala-Burgos ${ }^{\mathrm{a}}$, Francisco Javier Solorio-Sánchez ${ }^{\mathrm{a}}$, Carlos Fernando Aguilar-Pérez ${ }^{\mathrm{a}}$, \\ Juan Carlos Ku-Vera ${ }^{a}$
}

\begin{abstract}
RESUMEN
El objetivo fue evaluar el efecto de los taninos condensados (TC) contenidos en el follaje de arbóreas tropicales, sobre el consumo de materia seca y orgánica (CMS, CMO), la digestibilidad de la materia seca (DMS) y emisiones de metano (CH 4$)$ en ovinos alimentados con una ración basal de pasto Pennisetum purpureum. Cuatro ovinos de pelo con un peso vivo promedio de $21.6 \pm 2.0$ kg se sometieron a cuatro tratamientos en un diseño cuadro latino $4 \times 4$. Los tratamientos fueron $P$. purpureum (PP), $P$. purpureum + Havardia albicans (PPHA), P. purpureum + Bursera simaruba (PPBS), y P. purpureum + Acacia pennatula (PPAP); el follaje de las arbóreas se incluyó en $300 \mathrm{~g} / \mathrm{kg}$ de MS de la ración. El consumo, la digestibilidad y la producción total de metano entérico se midieron por periodos de $23 \mathrm{~h}$. La producción de metano se midió en cajas de respiración de circuito abierto. El consumo y digestibilidad de la MS y materia orgánica (MO) entre los tratamientos PP, PPHA, PPBS y PPAP no se afectaron por la inclusión de los follajes de arbóreas en la ración ( $P>0.05$ ). Cuando la producción de $\mathrm{CH}_{4}$ se expresó en $\mathrm{L} / \mathbf{k g}$ del $\mathrm{CMS}, \mathrm{CMO}$ o consumo de fibra detergente neutro los resultados fueron similares entre tratamientos (PP, PPHA, PPBS y PPAP) $(\boldsymbol{P}>\mathbf{0 . 0 5})$. Se concluye que los TC contenidos en el follaje de Havardia albicans, Acacia pennatula y Bursera simaruba no afectaron las emisiones de $\mathrm{CH}_{4}$ al nivel de incorporación de 30 \% de la MS; tampoco se observaron efectos en el CMS, CMO, DMS y concentración molar de ácidos grasos volátiles.
\end{abstract}

PALABRAS CLAVE: Arboles tropicales, Metano, Ovinos de pelo, Taninos condensados, Ácidos grasos volátiles.

\begin{abstract}
The aim of the work was to evaluate the effect of condensed tannins (CT) containing in the foliage's on dry matter intake and organic matter (DMI and OMI), dry matter digestibility (DMD) and methane $\left(\mathrm{CH}_{4}\right)$ emissions in sheep fed a basal ration of Pennisetum purpureum grass. Four hair sheep with an average live weight of $21.6 \pm 2.0 \mathrm{~kg}$ were allotted to four treatments in a $4 \mathrm{x}$ 4 latin square design. The treatments were $P$. purpureum (PP), P. purpureum + Havardia albicans (PPHA), P. purpureum + Bursera simaruba (PPBS), and purpureum + Acacia pennatula (PPAP), the foliage of tree was included $300 \mathrm{~g} / \mathrm{kg}$ DM of the ration. Intake, digestibility of DM and total methane emissions (L/d) were recorded for periods of $23 \mathrm{~h}$ when sheep were housed in open-circuit respiration head boxes. Intake and digestibility of the dry matter (DM) and organic matter (OM) between treatments PP, PPHA, PPBS and PPAP, were not affected by inclusion the foliage's of trees in the ration $(P>0.05)$. When the production of methane was expressed in L/ kg of DMI, OMI or neutral detergent fiber intake, the results were similar between treatments (PP, PPHA, PPBS, PPAP) ( $P>0.05)$. It is concluded that CT contained in the foliage of Havardia albicans, Acacia pennatula y Bursera simaruba not affected the $\mathrm{CH}_{4}$ emissions an levels of incorporation as $30 \%$ of ration DM, since there are no effects on DMI, OMI, DMD and molar concentration of volatile fatty acids.
\end{abstract}

KEY WORDS: Tropical trees, Methane, Hair sheep, Condensed tannins, Volatile fatty acids.

\footnotetext{
Recibido el 23 de marzo de 2016. Aceptado el 26 de abril de 2016.

${ }^{a}$ Facultad de Medicina Veterinaria y Zootecnia, Universidad Autónoma de Yucatán. 97300 Mérida, Yucatán, México.

${ }^{b}$ Instituto Tecnológico de la Zona Maya, Ejido Juan Sarabia. México.

'División Académica de Ciencias Agropecuarias, Universidad Juárez Autónoma de Tabasco. México.

${ }^{d}$ El Colegio de la Frontera Sur (ECOSUR), Ganadería y Ambiente. San Cristóbal de Las Casas, Chiapas. México.

*autor de correspondencia: pineiroiamc@gmail.com
} 


\section{INTRODUCCIÓN}

El metano $\left(\mathrm{CH}_{4}\right)$ es uno de los principales gases de efecto invernadero subproducto de la fermentación de los carbohidratos en el rumen ${ }^{(1)}$. En este sentido, los rumiantes contribuyen con el $40 \%$ de las 80 millones de toneladas de $\mathrm{CH}_{4}$ que se emiten por actividades antropogénicas globales ${ }^{(2)}$; adicionalmente a su efecto en el calentamiento global, el $\mathrm{CH}_{4}$ representa entre el 2 y $12 \%$ de la energía total consumida por los rumiantes ${ }^{(3)}$.

Aunado a esto, en las regiones tropicales los rumiantes son alimentados con forrajes de baja calidad; con una concentración de proteína cruda $(<7 \%)$, digestibilidad $(<50 \%)$ y alta proporción de carbohidratos estructurales (60 a $80 \%$ de FDN); estos factores favorecen un patrón de fermentación acética y una menor tasa de pasaje, la cual aumenta la síntesis y producción de $\mathrm{CH}_{4}$ ruminal ${ }^{(4,5)}$ así como un mejor desempeño productivo.

Sin embargo, en las regiones tropicales existe una gran abundancia de leguminosas que contienen taninos condensados (TC) con capacidad de reducir la población y actividad de los protozoarios ${ }^{(6)}$ la población de Archaeas metanogénicas y las emisiones de $\mathrm{CH}_{4}{ }^{(7)}$, o mejorando el comportamiento animal (mayor ganancia de peso y producción de leche) como una estrategia en la reducción de la producción de $\mathrm{CH}_{4}$.

El objetivo de este trabajo fue determinar el efecto de los TC contenidos en el follaje de arbóreas tropicales en ovinos de pelo alimentados con Pennisetum purpureum, sobre el consumo, digestibilidad, la producción de $\mathrm{CH}_{4}$, y el patrón de fermentación ruminal.

\section{MATERIAL Y MÉTODOS}

Los animales se manejaron de acuerdo con las normas de bienestar animal de la Facultad de Medicina Veterinaria y Zootecnia de la Universidad Autónoma de Yucatán. El trabajo se realizó en esta Facultad, ubicada en Mérida, Yucatán $\left(21^{\circ} 15^{\prime} \mathrm{N} 83^{\circ}\right.$ 32 'W), México. El clima de la región es de tipo AW0, considerado como cálido subhúmedo con precipitación media anual de $984.4 \mathrm{~mm} \mathrm{y}$ temperatura media anual de $26.8^{\circ} \mathrm{C}$.
Se utilizaron cuatro ovinos Pelibuey con peso promedio de $21 \pm 2 \mathrm{~kg}$, alojados en jaulas metabólicas, dentro de un edificio techado, con piso de cemento y desprovisto de paredes. Antes de iniciar la fase experimental los animales se desparasitaron internamente con Ivermectina $\AA$ al $1 \% ; 1 \mathrm{ml}$ por cada $50 \mathrm{~kg}$ de peso vivo (PV) y se les aplicó vitaminas ADE (1 ml por cada $10 \mathrm{~kg}$ de PV).

Se utilizó un diseño en cuadro latino $(4 \times 4)^{(8)}$; cada periodo tuvo una duración de 18 días (13 días para la adaptación al manejo y a la dieta y 5 para la medición de las variables propuestas).

La dieta base consistió en forraje verde picado de pasto Taiwán (Pennisetum purpureum) más la inclusión $30 \%$ de materia seca (MS) $(300 \mathrm{~g} / \mathrm{kg}$ de MS) como follaje de Havardia albicans, Acacia pennatula o Bursera simaruba cuya composición química se muestra en el Cuadro 1. Los tratamientos experimentales fueron pasto (PP): P. purpureum, (PPHA): P. purpureum más $30 \%$ del follaje de Havardia albicans, (PPBS): P. purpureum más $30 \%$ Bursera simaruba o (PPAP): P. purpureum más Acacia pennatula. Las arbóreas se incluyeron como fuente de TC en la ración; adicionalmente los animales se suplementaron con sales minerales Fogysal ovino $®(100 \mathrm{~g} /$ animal/día).

Cuadro 1. Composición química de las arbóreas y pasto utilizado en los tratamientos

\begin{tabular}{lcccc}
\hline & \multicolumn{4}{c}{ Composición química (\% de la MS) } \\
\cline { 2 - 5 } Variables & PP & HA & BS & AP \\
\hline MS & 23.3 & 46.0 & 28.5 & 46.0 \\
MO & 93.8 & 94.4 & 91.4 & 94.9 \\
FDN & 67.9 & 41.1 & 52.1 & 40.5 \\
FDA & 41.2 & 36.0 & 32.1 & 24.1 \\
PC & 6.8 & 14.4 & 13.9 & 13.9 \\
FTa $^{\text {a }}$ & 0.0 & 3.5 & 1.8 & 8.0 \\
TC $^{b}$ & 0.0 & 22.0 & 13.3 & 18.9 \\
\hline
\end{tabular}

$\mathrm{PP}=$ Pennisetum Purpureum; $\mathrm{HA}=$ Havardia albicans; $\mathrm{BS}=$ Bursera simaruba; $\mathrm{AP}=$ Acacia pennatula.

$\mathrm{MS}=$ materia seca: $\mathrm{MO}=$ materia orgánica: $F D N=$ fibra detergente neutro; FDA= fibra detergente acida; $\mathrm{PC}=$ proteína cruda; $\mathrm{FT}=$ fenoles totales; $\mathrm{TC}=$ taninos condensados. aExpresado en gramos de ácido tánico eq/kg MS. ${ }^{b}$ Expresado en aramos de leucocianidina ea/ka MS. 


\section{Variables evaluadas}

Determinación del $\mathrm{pH}$ y patrón de ácidos grasos volátiles (AGV)

Se tomaron muestras de líquido ruminal vía sonda esofágica de acuerdo a lo descrito por RamosMorales et $a{ }^{(9)}$ durante los cinco días de mediciones en cada periodo, a las $6 \mathrm{~h}$ posteriores a la alimentación, con el fin de determinar el pH y AGV.

El pH se midió inmediatamente, después de obtenida la muestra de líquido ruminal se filtró con gazas dobles, y posteriormente con un potenciómetro portátil (HANNA ${ }^{\circledR}$ instruments, Woonsocket, USA), calibrado con buffers de referencia de pH 4, 7 y 10 , se registró el pH previo a la obtención de una muestra para determinar AGV. Para el análisis de AGV se tomaron $4 \mathrm{ml}$ de líquido ruminal y se conservaron en $1 \mathrm{ml}$ de solución desproteinizante compuesta por ácido metafosfórico y ácido 3-methilvalerico.

Para la determinación de los AGV se empleó la técnica propuesta por Ryan ${ }^{(10)}$ usando un cromatógrafo de gases (Hewlett-Packard, 5890 serie III), equipado con un detector de ionización de flama (FID); el tipo de columna utilizada fue HPFFAP de $30 \mathrm{~m} \times 0.53 \mathrm{~mm}$, la temperatura del inyector fue de $200^{\circ} \mathrm{C}$ y la del detector de $200^{\circ} \mathrm{C}$.

\section{Medición de la producción de $\mathrm{CH}_{4}$}

La medición de $\mathrm{CH}_{4}(\mathrm{~L} / \mathrm{d})$ entérico se realizó por medio de cajas de respiración de circuito abierto(11), con dimensiones de $1.10 \mathrm{~m} \times 0.40 \mathrm{~m} \times 0.30 \mathrm{~m}$, (alto, ancho y largo, respectivamente) con un volumen interno de $0.132 \mathrm{~m}^{3}$.

Las cajas de respiración se construyeron con paneles de acrílico transparente de $9 \mathrm{~mm}$ pegados a angulares de 2 pulgadas y selladas con poliuretano (Construflex $\AA$, Multiaccesorios Monterrey SA de $\mathrm{CV}$ ), la entrada de la cabeza del animal se selló con tela de mezclilla. El aire se extrajo mediante una bomba de vacío (Sable Systems International, Las Vegas, USA); ésta produjo una fuerza de vacío de 125 pascales registrado con manómetros de presión diferencial (Heavy Duty Series 407910, Extech Instruments Corporation, USA).

Los datos fueron extraídos y ajustados mediante el programa Expedata ${ }^{\circledR}$ (Sable Systems International, Las Vegas, USA). La medición de la concentración de metano se realizó alternativamente en las dos cajas de respiración, empleando 25 min en cada una. La producción de $\mathrm{CH}_{4}$ se calculó con la producción media por unidad de tiempo y flujo.

El metano se midió por medio de un analizador (MA-10, Sable Systems International Inc., las Vegas, USA) que utiliza el método de infrarrojo, con una tasa de recuperación del $99.999 \%{ }^{(11)}$ calibrado con $\mathrm{N}_{2}$ y $\mathrm{CH}_{4}$ en nitrógeno con una concentración de 1,000 ppm (Praxair, USA).

Los animales se alojaron dentro de las cajas de respiración por un periodo de $6 \mathrm{~h}$, y las mediciones se realizaron durante dos días consecutivos ${ }^{(11)}$. Para el cálculo de la producción total de $\mathrm{CH}_{4}$, los valores obtenidos se ajustaron a $24 \mathrm{~h}$, utilizando el programa Expedita (Sable Systems, las Vegas, USA).

\section{Consumo voluntario}

La alimentación fue a libre acceso, si los animales no dejaban rechazo se les aumentaba el 15 $\%$ de lo ofrecido el día anterior. La dieta ofrecida cubrió un consumo estimado de MS de $800 \mathrm{~g} / \mathrm{d}$. Las unidades experimentales se alimentaron una vez al día ofreciéndoles el $100 \%$ de la ración a las $0900 \mathrm{~h}$. Los rechazos de alimento se retiraron a las $0900 \mathrm{~h}$ del día siguiente.

\section{Digestibilidad aparente}

La determinación de la digestibilidad aparente de la MS, MO y FDN se realizó mediante el método de recolección total de excremento ${ }^{(12)}$, tomando una muestra del $10 \%$ del total de las deposiciones producidas por día, en los últimos seis días del periodo experimental y se conservaron en congelación a $-4{ }^{\circ} \mathrm{C}$; se mezclaron por cada tratamiento y se tomaron submuestras del $10 \%$ para su análisis químico.

\section{Análisis químicos}

La determinación de MS de las muestras de forraje ofrecido se realizó en una estufa de aire forzado a $55{ }^{\circ} \mathrm{C}$ por $48 \mathrm{~h}$ (peso constante) $(\# 7.007)^{(13)}$. El contenido de $\mathrm{N}$ se realizó (CP; $\mathrm{N} \times$ 6.25) por combustión utilizando un equipo LECO CN2000 serie 3740 (LECO, Corporation, \#2.057) ${ }^{(13)}$. La MO se determinó por incineración en una mufla a $550{ }^{\circ} \mathrm{C}$ por $6 \mathrm{~h}$ (AOAC Method \#923.03) y el 
contenido de FND y FDA se determinó según lo sugerido por Van Soest et a ${ }^{\left({ }^{14}\right)}$. El contenido de taninos condensados (TC) se realizó por medio del método de HCL-Butanol(15).

\section{Análisis estadísticos}

Los datos de consumo voluntario, digestibilidad aparente, producción de metano y la concentración molar de AGV se sometieron a un análisis de varianza para un diseño cuadro latino $(4 \times 4)^{(8)}$ por medio del procedimiento PROC GLM del SAS(16). Las medias de los tratamientos se compararon con la prueba de Tukey con un alfa de 0.05 .

\section{RESULTADOS}

\section{Composición química}

El follaje de las arbóreas tropicales presenta una mejor calidad en cuanto a PC así como un contenido de FDN y FDA menor respecto a la gramínea ( $P$. purpureum). Los follajes de las arbóreas evaluadas en este estudio mostraron una concentración promedio de $18 \%$ de TC (Cuadro 1).

\section{Consumo voluntario}

El consumo de MS, MO, FDN y FDA (CMS, CMO, CFDN y CFDA) fue similar entre los tratamientos con un promedio de $492.8,462.12,188.44$ y 182.22 $\mathrm{g} /$ día respectivamente $(P>0.05)$. El consumo de PC ( $g / d i ́ a)$ fue similar en el tratamiento con PP respecto a los tratamientos PPHA y PPBS, sin embargo, se observó diferencia entre PP y PPAP $(P<0.05)$. El consumo de materia seca y orgánica digestible (CMSD y CMOD) fue similar entre los tratamientos, sin embargo, se observó diferencia entre PP vs PPHA, PPBS y PPAP en el consumo de FDND $(P<0.05)$ (Cuadro 2).

Cuadro 2. Consumo de materia seca y digestibilidad en ovinos alimentados con Pennisetum purpureum y follajes de árboles tropicales

\begin{tabular}{|c|c|c|c|c|c|c|}
\hline & \multicolumn{6}{|c|}{ Tratamientos 30\% de inclusión de arbóreas (300 g/kg de MS) } \\
\hline & PP & PPHA & PPBS & PPAP & $\mathrm{EE}$ & $P$ \\
\hline TC (g/kg de MS) & 0 & 66 & 39 & 57 & & \\
\hline Peso vivo, $\mathrm{kg}$ & 18.4 & 20.9 & 19.0 & 22.4 & 0.516 & \\
\hline \multicolumn{7}{|c|}{ Consumo voluntario } \\
\hline MS, g/día & 487.9 & 474.7 & 490.1 & 518.8 & 25.5 & 0.6140 \\
\hline MS, $\%$ del peso vivo & 2.4 & 2.2 & 2.5 & 2.2 & 0.1 & 0.1986 \\
\hline MS, g/kg 0.75 & 50.3 & 46.9 & 51.9 & 47.6 & 2.2 & 0.4237 \\
\hline MO, g/día & 456.5 & 447.5 & 455.4 & 489.1 & 23.4 & 0.5614 \\
\hline FDN, g/día & 171.2 & 181.8 & 201.2 & 199.7 & 18.5 & 0.3627 \\
\hline FDA, g/día & 198.9 & 182.5 & 174.6 & 172.9 & 15.5 & 0.2084 \\
\hline PC, g/día & $32.7^{\mathrm{b}}$ & $38.1^{\mathrm{ab}}$ & $42.0 \mathrm{ab}$ & $45.9^{a}$ & 2.4 & 0.0177 \\
\hline \multicolumn{7}{|c|}{ Digestibilidad aparente } \\
\hline MS, g/kg de MS & 632.1 & 738.2 & 677.6 & 713.3 & 34.7 & 0.0960 \\
\hline $\mathrm{MO}, \mathrm{g} / \mathrm{kg}$ de $\mathrm{MO}$ & 654.9 & 738.2 & 695.1 & 730.7 & 35.1 & 0.1040 \\
\hline FDN, g/kg de FDN & $319.4^{b}$ & $622.0^{a}$ & $558.3^{a}$ & $581.3^{a}$ & 66.4 & 0.0031 \\
\hline$P C, g / k g$ de $P C$ & 641.4 & 713.0 & 696.9 & 721.8 & 38.3 & 0.2452 \\
\hline \multicolumn{7}{|c|}{ Consumo de componentes digestibles* } \\
\hline MS, g/día & 312.8 & 352.4 & 333.1 & 371.0 & 26.0 & 0.1896 \\
\hline MO, g/día & 302.5 & 338.7 & 317.4 & 358.2 & 23.6 & 0.1610 \\
\hline
\end{tabular}

$\mathrm{PP}=$ Pennisetum purpureum; $\mathrm{HA}=$ Havardia albicans; $\mathrm{BS}=$ Bursera simaruba; $\mathrm{AP}=$ Acacia pennatula.

$\mathrm{TC}=$ taninos condensados; $\mathrm{MS}=$ materia seca; $\mathrm{MO}=$ materia orgánica; $\mathrm{FDN}=$ fibra detergente neutro: $\mathrm{EE}=$ error estándar.

*El consumo digestible de cada componente proviene de multiplicar el consumo voluntario por su digestibilidad.

ab Medias nor hilera con diferente literal indican diferencias $(P<0.05)$ 
Cuadro 3. Ácidos grasos volátiles (mmol/L) en ovinos alimentados con Pennisetum purpureum y follajes de árboles tropicales

\begin{tabular}{lcccccc}
\hline & \multicolumn{7}{c}{ Tratamientos 30\% de inclusión de arbóreas (300 g/kg de MS) } \\
\cline { 2 - 7 } TC (g/kg de MS) & PP & PPHA & PPBS & PPAP & EE & valor de $P$ \\
\cline { 2 - 6 } pH ruminal & 0 & 66 & 39 & 57 & \\
& 7.1 & 7.1 & 7.0 & 7.1 & 0.5 & 0.7316 \\
Totales & \multicolumn{7}{c}{ Condiciones del rumen } \\
Acético & 44.0 & 53.5 & 50.2 & 53.6 & 4.16 & 0.3995 \\
Propiónico & 26.9 & 37.8 & 34.6 & 37.4 & 4.6 & 0.3861 \\
Butírico & 11.6 & 11.6 & 10.6 & 10.0 & 1.0 & 0.3684 \\
Isobutírico & 3.2 & 3.4 & 3.6 & 3.7 & 0.4 & 0.7672 \\
Isovalérico & 0.4 & 0.2 & 1.2 & 1.6 & 0.6 & 0.6280 \\
Valérico & 0.3 & 0.2 & 0.3 & 0.6 & 0.2 & 0.7918 \\
Ac:Pr & 1.5 & 0.2 & 0.0 & 0.2 & 0.4 & 0.5456 \\
\hline
\end{tabular}

$\mathrm{TC}=$ taninos condensados; $\mathrm{PP}=$ Pennisetum Purpureum; $\mathrm{HA}=$ Havardia albicans; $\mathrm{BS}=$ Bursera simaruba; $\mathrm{AP}=\mathrm{Acacia}$ pennatula. Ac:Pr: relación acético propiónico; TC= taninos condensados; $E E=$ error estándar. $(P>0.05)$.

\section{Digestibilidad aparente}

La digestibilidad de la MS y MO (DIGMS y DIGMO) fue similar entre los tratamientos con un promedio de 690.30 y 704.73 , respectivamente. Sin embargo, la digestibilidad de la FDN fue menor $(P<0.05)$ en 94.74, 74.19, $81.99 \%$ en el tratamiento PP con respecto a los tratamientos PPHA, PPBS y PPAP.

\section{Concentración de ácidos grasos volátiles $\boldsymbol{y} \mathrm{pH}$}

El pH no se afectó por la inclusión y la especie de las arbóreas tropicales ( $P>0.05)$. La concentración molar (mmol/L) de los AGV (acético, propiónico, butírico, valérico, isobutírico e isovalérico) no fue afectado $(P>0.05)$ por los tratamientos PP, PPHA, PPBS y PPAP, encontrándose promedios en la concentración molar de 34.1, 10.9, $3.4,0.4,0.8$ y 0.3 , respectivamente (Cuadro 3 ).

\section{Producción de metano entérico}

La producción de $\mathrm{CH}_{4}$ entérico expresado en L/día, L/kg de MSC, MOC y FDNC fue similar
( $P>0.05$ ) entre tratamientos PP, PPHA, PPBS y PPAP encontrando promedios de $17.8,36.8$, 39.3 y 100 , respectivamente (Cuadro 4 ).

\section{DISCUSIÓN}

\section{Composición química}

La composición química del follaje de las leguminosas fue similar a lo reportado en regiones tropicales ${ }^{(17)}$, teniendo una mayor concentración de PC y una menor cantidad de fracciones fibrosas con mayor digestibilidad( ${ }^{(4)}$. La cantidad de TC contenidos en las arbóreas (130 a $220 \mathrm{~g} / \mathrm{kg}$ de MS) es superior a otras arbóreas y plantas utilizadas comúnmente en la alimentación de rumiantes; por ejemplo Leucaena leucocephala, en sus diferentes variedades, Gliricidia sepium, Piscidia piscipula, Lespedeza cuneata y Lespedeza striata ${ }^{(6,7)}$.

\section{Consumo voluntario}

La inclusión de TC en las raciones en dosis superiores $>7 \%$ de TC de la MS o con la inclusión mayor al $30 \%$ del follaje de las arbóreas como parte 
Cuadro 4. Producción de $\mathrm{CH}_{4}$ en ovinos alimentados con Pennisetum purpureum y follajes de árboles tropicales

\begin{tabular}{lcccccc}
\hline & \multicolumn{7}{c}{ Tratamientos 30\% de inclusión de arbóreas (300 g/kg de MS) } \\
\cline { 2 - 7 } Variables & PP & PPHA & PPBS & PPAP & EE & $P$ \\
\cline { 2 - 7 } $\mathrm{TC}(\mathrm{g} / \mathrm{kg}$ de MS) & 0 & 66 & 39 & 57 & & \\
\hline & \multicolumn{7}{c}{ Producción de metano entérico } & & & \\
$\mathrm{L} \mathrm{CH} /$ día & 17.6 & 20.9 & 13.1 & 19.8 & 3.0 & 0.4797 \\
$\mathrm{~L} \mathrm{CH} / \mathrm{kg}$ de MSC & 37.7 & 44.2 & 26.9 & 38.7 & 7.0 & 0.4414 \\
$\mathrm{~L} \mathrm{CH} / \mathrm{kg}$ de MOC & 40.2 & 46.9 & 29.0 & 41.1 & 7.4 & 0.4637 \\
$\mathrm{~L} \mathrm{CH} / \mathrm{kg}$ de FDNC & 117.9 & 116.3 & 65.7 & 104.0 & 22.8 & 0.3083 \\
\hline
\end{tabular}

$\mathrm{TC}=$ taninos condensados; $\mathrm{PP}=$ Pennisetum Purpureum; $\mathrm{HA}=$ Havardia albicans; $\mathrm{BS}=$ Bursera simaruba; $\mathrm{AP}=$ Acacia pennatula; $\mathrm{EE}=$ error estándar.

$\mathrm{MSC}=$ materia seca consumida; $\mathrm{MOC}=$ materia orgánica consumida; $F D N C=$ fibra detergente neutro consumida.

$(P>0.05)$.

de la ración, puede reducir el consumo voluntario y la digestibilidad de los componentes de la ración ${ }^{(18)}$; sin embargo, en este estudio con la inclusión $30 \%$ de las arbóreas no se observó efecto sobre el consumo de MS, MO, FDN y FDA, aunque las arbóreas proporcionaban una menor cantidad de FDN y FDA (Cuadros 1 y 2 ).

El consumo de alimento no se afectó posiblemente porque los ovinos poseen proteínas salivales capaces de bloquear el efecto de los TC, reduciendo la astringencia y su efecto sobre la digestibilidad, con lo cual el tiempo de permanencia en el rumen sería prolongado. Por otra parte, es posible que los ovinos seleccionaran de la mezcla ofrecida las fracciones sin TC consumiendo en mayor proporción el pasto ${ }^{(19)}$. Resultados similares han sido encontrados con la suplementación de ovinos y cabras con la inclusión de $30 \%$ de diferentes arbóreas que contienen $\mathrm{TC}^{(7)}$.

En este estudio, la inclusión del $30 \%$ del follaje de arbóreas en la dieta de ovinos mejoró la calidad de la dieta; resultados similares han sido encontrados en otros estudios ${ }^{(5,20)}$, observando que la suplementación con follajes arbóreos incrementa el consumo de PC debido al mayor aporte de las arbóreas ( $140 \mathrm{~g} / \mathrm{kg}$ de MS), además de su bajo contenido de FDN y FDA, lo cual aumenta la tasa de pasaje incrementando con ello el consumo de $\mathrm{PC}^{(21)}$, a diferencia de cuando los animales son alimentados con forrajes de baja calidad (PC <7 \%) y alto contenido de fibra ( $67.9 \%)$ lo cual aumenta el tiempo de permanencia en el rumen por efecto del llenado gástrico, reduciendo el consumo voluntario de nutrientes ${ }^{(5)}$.

Por otra parte, el elevado consumo de PC en los tratamientos con arbóreas (28\% superior al tratamiento control) pudo haber generado un incremento en la cantidad de nitrógeno excretado en la orina debido al desbalance entre energía y proteína en la dieta.

\section{Digestibilidad aparente}

En este estudio, con el uso de follaje de las arbóreas que contienen TC en concentraciones de 130 a $220 \mathrm{~g} / \mathrm{kg}$ de MS, cantidades superiores al de otras arbóreas utilizadas comúnmente en la alimentación de rumiantes (Leucaena leucocephala, Piscidia piscipula y Gliricidia sepium), se observó que la inclusión de $30 \%$ del follaje de estas arbóreas como parte de la MS de la ración, no afectó la digestibilidad de la MS y MO.

Sin embargo, se observó un incremento en la digestibilidad de la FDN y PC cuando se incluyen follajes de arbóreas tropicales en comparación del tratamiento basado en $P$. purpureum; esto se debe a la alta concentración de carbohidratos estructurales (FDN 67.9 \%) que estas gramíneas poseen ${ }^{(4,21)}$, 
diferente al de los follajes arbóreos, los cuales contienen cantidades menores de FDN (44.5\% de la MS), lo que hace que sean más susceptibles al ataque de los microorganismos ruminales encargados de la digestión, degradación y fermentación de la fibra.

Por otra parte, el aporte de PC es superior al de las gramíneas, con lo que provee las condiciones

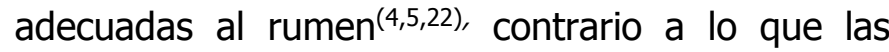
gramíneas pueden aportar durante la época de secas $(6.5 \%$ de $\mathrm{PC})$, con lo cual no se cubren los requerimientos mínimos ( $7 \%$ de $\mathrm{PC}$ ) para la función y crecimiento adecuado de los microorganismo del rumen.

La variación del efecto de los TC es inconsistente y depende del tipo de TC, su estructura, su peso molecular, la dosis, la fuente y la especie animal utilizada( ${ }^{(23,24)}$; en este sentido, se ha documentado que los pequeños rumiantes son capaces de desactivar a los $\mathrm{TC}^{(19)}$ evitando la reducción de la población de protozoarios responsables de la degradación de la fibra hasta del $50 \%(25)$, además de evitar la formación de complejos entre carbohidratos y proteínas, lo cual reduciría la digestibilidad de los componentes de la ración.

\section{pH y proporción molar de los AGV}

En las raciones evaluadas se encontró un $\mathrm{pH}$ promedio de 7.0 típico de una fermentación acética, debido a una dieta basada en celulosa, el cual mantiene el pH similar al encontrado en otros estudios cuando se incluyen arbóreas tropicales a una dieta basada en pastos. Este $\mathrm{pH}$ registrado demuestra que no hubo cambios en los perfiles de AGV con o sin la inclusión del follaje arbóreo.

Los cambios en el patrón de fermentación ruminal dependen principalmente del tipo de dieta y de las poblaciones bacterianas; algunos estudios indican que los TC tiene la capacidad de cambiar en el patrón y concentración de los $\mathrm{AGV}^{(26)}$. En este estudio no se observaron cambios en la concentración total e individual de los AGV, resultados apoyados por Rira et a ${ }^{(27)}$ quienes con la inclusión de $44 \%$ de $L$. leucocephala, no observaron efectos sobre los AGV.
Los resultados encontrados en estos estudios mostraron que los TC no afectaron el pH, la concentración y proporción de AGV, lo que sugiere que la inclusión de arbóreas con TC no generó cambios en las poblaciones bacterianas y protozoarias del rumen, además no modificaron la digestión de la MS y MO.

Sin embargo, Tan et $a^{(26)}$ encontraron una reducción de la concentración de ácido propiónico con la inclusión de TC de L. leucocephala, mientras que otros estudios in vitro demuestran la capacidad de los TC de aumentar la concentración de ácido propiónico ${ }^{(28)}$. Es posible que la dosis de inclusión de los TC usando como fuente las arbóreas tropicales no sean suficientes para cambiar el patrón de fermentación y la población bacteriana ruminal ${ }^{(9,15,25)}$.

\section{Producción de metano}

La producción de $\mathrm{CH}_{4}(36.8 \mathrm{~L} / \mathrm{kg}$ de MS) fue similar a reportes ${ }^{(4,23)}$ en ovinos alimentados con forrajes y follajes de arbóreas tropicales. Sin embargo, no se observó efecto de la inclusión de las arbóreas que contienen $\mathrm{TC}$, sobre la producción de $\mathrm{CH}_{4}$; estos resultados difieren a lo encontrado por Tiemann et $a^{(23)}$ quienes con la inclusión de $30 \%$ de arbóreas que contenían $\mathrm{TC}$, redujeron la producción de $\mathrm{CH}_{4}$ entérico.

Por su parte investigadores ${ }^{(29)}$ utilizando la inclusión de $L$. leucocephala en la dieta de ovinos, redujeron en $26 \%$ la cantidad de energía en forma de $\mathrm{CH}_{4}$, resultados apoyados por Soltan et $a^{(7)}$ quienes reportan disminución en $14 \%$ la producción de $\mathrm{CH}_{4}$. En este sentido bajo condiciones in vitro se ha encontrado que la inclusión de TC reduce la producción de $\mathrm{CH}_{4}{ }^{(26)}$.

En este sentido, diversos estudios han demostrado que la inclusión de TC usando como fuente alguna arbórea, reducen las emisiones de $\mathrm{CH}_{4}$ entérico ${ }^{(4,5,6)}$ debido a su efecto en la reducción de las poblaciones protozoarias y bacterias metanogénicas (efecto directo) o a través de la reducción de la degradación y digestión de los componentes de la ración (efecto indirecto)(22,23,30).

En este estudio, no se observó tal efecto sobre la producción de $\mathrm{CH}_{4}$, esto puede ser debido a la 
naturaleza de alimentación de los ovinos, los cuales están adaptados al consumo de plantas ricas en TC, los cuales generan mecanismos de resistencia (bloqueadores salivales) o de selección $^{(19)}$, con lo cual reducen la actividad o el consumo de los TC, evitando la formación de complejos entre TC, proteínas y carbohidratos.

Por otra parte, el aumento del aporte de PC así como mayor digestibilidad de la MS y FDN de las dietas con la inclusión del follaje de arbóreas tropicales, pudo mejorar las condiciones del rumen aumentando la degradación y fermentación de la $\mathrm{MO}$, lo que pudo incrementar la concentración de los hidrógenos libres $(\mathrm{H})$, sustrato utilizado por las bacterias metanogénicas para la reducción del $\mathrm{CO}_{2}$ y formación de $\mathrm{CH}_{4}{ }^{(30)}$.

El aumento en la calidad de la dieta cuando se hace uso del recurso arbóreo podría aumentar la ganancia de peso en ovinos (eficiencia alimenticia), lo cual sería una forma indirecta en la reducción de las emisiones de $\mathrm{CH}_{4}$ (litros de metano/kg de peso ganado).

\section{CONCLUSIONES E IMPLICACIONES}

Se concluye que la inclusión del $30 \%$ de las arbóreas tropicales Havardia albicans, Bursera simaruba y Acacia pennatula como fuente de TC, no afectó el consumo, fermentación ruminal y las emisiones de $\mathrm{CH}_{4}$; sin embargo, mejoró el aporte de proteína y la digestibilidad de la MS, PC y FDN. Es posible que se necesite incrementar el nivel de inclusión en la dieta de ovinos para observar algún cambio en la producción de $\mathrm{CH}_{4}$.

\section{AGRADECIMIENTOS}

El primer autor agradece a CONACYT-México, por haber otorgado la beca para estudios de doctorado en la Facultad de Medicina Veterinaria y Zootecnia de la Universidad Autónoma de Yucatán, Mérida, México.

\section{LITERATURA CITADA}

1. Bodas R, Prieto N, García-González R, Andrés S, Giráldez FJ, López S. Manipulation of rumen fermentation and methane production with plant secondary metabolites. Anim Feed Sci Technol 2012;(176):78-93.

2. Gerber PJ, Steinfeld H, Henderson B, Mottet A, Opio C, Dijkman J, et al. Tackling climate change through livestock - A global assessment of emissions and mitigation opportunities. Food and Agriculture Organization of the United Nations (FAO), Rome. 2013.

3. Moss AR, Jouany JP, Newbold J. Methane production by ruminants: its contribution to global warming. Ann Zootec 2000;(49):231-253.

4. Archimède $H$, Eugène $M$, Marie-Magdeleine $C$, Boval $M$, Martin $C$, Morgavi DP, et al. Comparison of methane production between $\mathrm{C} 3$ and C4 grasses and Legumes. Anim Feed Sci Technol 2011;(166167):59- 64.

5. Kennedy PM, Charmley E. Methane yields from Brahman cattle fed tropical grasses and legumes. Anim Prod Sci 2012;(52):225-239.

6. Animut G, Puchala $R$, Goetsch $A L$, Patra $A K$, Sahlu $T$, Varel $V H$, Wells J. Methane emission by goats consuming diets with different levels of condensed tannins from lespedeza. Anim Feed Sci Technol 2008;(144):212-227.

7. Soltan, YA, Morsy AS, Sallam SMA, Lucas RC, Louvandini H, Kreuzer $M$, Abdalla AL. Contribution of condensed tannins and mimosine to the methane mitigation caused by feeding Leucaena leucocephala. Arch Anim Nutr 2013;(67):169-184.

8. Cochran WG, Cox GM. Diseños experimentales. $2^{a}$ ed. México: Trillas; 1991.

9. Ramos-Morales E, Arco-Pérez A, Martín-García AI, Yáñez-Ruiz DR, Frutos P, Hervás G. Use of stomach tubing as an alternative to rumen cannulation to study ruminal fermentation and microbiota in sheep and goats. Anim Feed Sci Technol 2014;(198):57-66.

10. Ryan JP. Determination of volatile fatty acids and some related compounds in ovine rumen fluid, urine and blood plasma by gasliquid chromatography. Annal Biochem 1980;(108):374-384.

11. Pinares-Patiño C, Garry W. Technical manual on respiration chamber designs. Ministry of Agriculture and Forestry. ISBN 978-0478-38788-9 (on line) New Zeland. 2011.

12. Schneider BH, Flatt WP. The evaluation of feeds through digestibility experiments. Athens: The University of Georgia Press; 1975.

13. AOAC. Official methods of analysis. Association of Official Analytical Chemists. 15th. ed, Washington DC, USA. 1980.

14. Van Soest PJ, Robertson JB, Lewis BA. Methods for dietary fiber, neutral detergent fiber and non-starch polysaccharides in relation to animal nutrition. J Dairy Sci 1981;(74):3583.

15. Makkar HPS. Quantification of tannins in tree and shrub foliage: a laboratory analysis. Dordrecht, The Netherlands: Kluwer Amademic Publisher; 2003.

16. SAS. Institute Inc., SAS/STAT. Software, Ver. 9.00. SAS, Cary, NC, USA. 2006.

17. Ku-Vera J, Ramírez C, Jiménez G, Alayón J, Ramírez L. Árboles y arbustos para la producción animal en el trópico mexicano. Conferencia electrónica. FAO. http://www.fao.org/livestock/agap/ frg/agrofor1/ku10.htm.

18. Barry TN, Duncan SJ. The role of condensed tannins in the nutritional value of Lotus pedunculatus for sheep. $\mathrm{Br} \mathrm{J}$ Nutr 1984;(51):485-491. 
19. Alonso-Díaz MA, Torres-Acosta JFJ, Sandoval-Castro CA, Hoste H. Tannins in tropical tree fodders to small ruminants: A friendly foe? Small Ruminant Res 2010;(89):164-173.

20. Burke JM, Miller JE, Terrill TH, Mosjidis JA. The effects of supplemental Sericea lespedeza pellets in lambs and kids on growth rate. Livest Sci 2014;(159):29-36.

21. Assoumaya $C$, Sauvant $D$, Archimède $H$. Etudecomparative de l'ingestion et de la digestion des fourragestropicaux et tempérés. INRA. Prod Anim 2007;(20):383-392.

22. Delgado DC, Galindo J, Ibett JCO, Dominguez M, Dorta N. Suplementación con follaje de L. leucocephala. Su efecto en la digestibilidad aparente de nutrientes y producción de metano en ovinos. Rev Cub Cien Agr 2013;(47):267-271.

23. Tiemann $T$, Lascano CE, Wettstein HR, Mayer AC, Kreuzer M, Hess HD. Effect of the tropical tannin-rich shrub legumes Calliandra calothyrsus and Flemingia macrophylla on methane emission and nitrogen and energy balance in growing lambs. Animal 2008;(2):790-799.

24. Naumann HD, Tedeschi LO, Muir JP, Lambert BD, Kothmann MM. Effect of molecular weight of condensed tannins from warm-season perennial legumes on ruminal methane production in vitro. Biochem Syst Ecol 2013;(50):154-162.
25. Coleman GS. The distribution of carboxymethyl cellulase between fractions taken from the rumen of sheep containing no protozoa or one of five different protozoal populations. J Agr Sci 1986;(106):121-127.

26. Tan HY, Sieo CC, Abdullah N, Liang JB, Huang, XD, Ho YW. Effects of condensed tannins from Leucaena on methane production, rumen fermentation and populations of methanogens and protozoa in vitro. Anim Feed Sci Technol 2011;(169):185-193.

27. Rira $M$, Morgavi DP, Archimède $H$, Marie-Magdeleine $C$, Popova $M$, Bousseboua $H$, Doreau $M$. Potential of tannin-rich plants for modulating ruminal microbes and ruminal fermentation in sheep. J Anim Sci 2015;(93):334-347.

28. Hassanat F, Benchaar C. Assessment of the effect of condensed (acacia and quebracho) and hydrolysable (chestnut and valonea) tannins on rumen fermentation and methane production in vitro. J Sci Food Agr 2013;(93):32-339.

29. Osakwe II, Steingass H. Ruminal fermentation and nutrient digestion in West African Dwarf (WAD) sheep fed Leucaena leucocephala supplemental diets. Agroforest Syst 2006;(67):129133.

30. Goel G, Makkar HPS. Methane mitigation from ruminants using tannins and saponins. Trop Anim Health Prod 2012;(44):729-739. 